Cahiers de la recherche sur les droits fondamentaux

Recherche sur les Droits Fondamentaux

11 | 2013

Le droit de la famille en (r)évolutions

\title{
La vie familiale des étrangers
}

\section{Catherine-Amélie Chassin}

\section{OpenEdition}

\section{Journals}

Édition électronique

URL : https://journals.openedition.org/crdf/4660

DOI : $10.4000 /$ crdf.4660

ISSN : 2264-1246

Éditeur

Presses universitaires de Caen

Édition imprimée

Date de publication : 1 novembre 2013

Pagination : 77-85

ISSN : 1634-8842

\section{Référence électronique}

Catherine-Amélie Chassin, «La vie familiale des étrangers », Cahiers de la recherche sur les droits

fondamentaux [En ligne], 11 | 2013, mis en ligne le 01 décembre 2014, consulté le 14 novembre 2022. URL : http://journals.openedition.org/crdf/4660 ; DOI : https://doi.org/10.4000/crdf.4660 


\title{
La vie familiale des étrangers
}

\author{
Catherine-Amélie CHASSIN \\ Maître de conférences en droit public à I'Université de Caen Basse-Normandie \\ Centre de recherche sur les droits fondamentaux et les évolutions du droit (CRDFED, EA 2132)
}

I. Les contours de la vie familiale

A. Une conception large de la famille

B. Des conceptions restrictives de la famille

II. Les droits attachés à la vie familiale de l'étranger
A. Le lieu de la vie familiale
B. La normalité de la vie familiale

La notion de vie familiale est par nature une notion polymorphe, propre à chaque société. Chaque communauté, chaque État, a progressivement construit sa propre notion de famille, déterminant ses contours, lui reconnaissant un certain nombre de droits dont les contenus évoluent en fonction des mutations sociétales. La vie familiale et son corollaire, le droit de la famille, pourraient presque apparaître pour ce qu'ils ne sont pas toujours, à savoir un long fleuve tranquille. Pourtant, il est bien souvent des pavés qui viennent tourmenter les eaux calmes - les débats échauffés entourant, en France, l'adoption de la loi étendant le mariage et l'adoption aux couples homosexuels en sont un excellent exemple.

Pour autant il n'est pas besoin de songer aux réformes d'envergure pour constater l'existence de troubles : ceuxci arrivent bien souvent du simple fait du franchissement d'une frontière. Le migrant, nouvel arrivant au sein d'une population, doit alors s'adapter aux mœurs et règles de cette population, se fondre dans le cadre social et se plier à ses règles de droit. Si l'on n'imagine guère de différence entre la vie familiale menée de part et d'autre du Rhin ou des Pyrénées, en revanche le fossé peut être d'autant plus important que les modes de vie d'origine divergent. Ce que le migrant tiendra pour sa propre conception de la vie familiale, une conception qu'il a toujours connue et dans laquelle il a grandi, pourra être fondamentalement remise en cause par les valeurs de son pays d'accueil. La vie familiale d'origine devra alors s'insérer dans ce nouveau cadre, faire des concessions majeures, parfois disparaître dans sa forme originelle. On pense immédiatement à ces hommes polygames, qui n'ont jamais connu d'autre modèle que la polygamie, ont toujours cru que le nombre d'épouses était proportionnellement égal à la puissance économique, et qui brusquement prennent conscience que la France n'admet, sur son territoire, que la monogamie; et qu'en conséquence les épouses surabondantes ne pourront suivre le migrant. Si la solution s'impose naturellement pour un Occidental, elle est loin d'être évidente pour celui qui vient d'un pays reconnaissant la polygamie. Et l'exemple n'est pas isolé: que penser d'un couple musulman ayant élevé un enfant comme le sien, et auquel brusquement on apprend que le mode de garde dudit enfant n'équivalant pas à une adoption, il ne peut les suivre? On le voit, les exemples sont en réalité nombreux, de ce bouleversement familial qu'induit la migration.

Quelques éléments émergent d'entrée de jeu, en particulier la question de savoir qui sont, exactement, ces étrangers. Le droit français les définit de façon très succincte, comme étant toute personne n'ayant pas la 
nationalité française ${ }^{1}$, ce qui renvoie en réalité à une catégorisation trop vaste pour être utilisable. Car la question reste ouverte des cas particuliers, tels les citoyens européens, les réfugiés et apatrides, ou le cas échéant la distinction (à faire ou non) selon la régularité du séjour au regard de la législation française. Dans le cadre de la présente étude, néanmoins, la notion sera appréhendée, sauf explicitation particulière, dans le cadre du droit commun.

Dans la même veine, on ne peut que constater l'étendue de la notion même de vie familiale, consacrée par l'article 8 de la Convention européenne des droits de l'homme (ciaprès Convention EDH), et déduite par la jurisprudence interne de l'article 9 du Code civil, lequel consacre le droit à la vie privée. Pour autant, vie privée et vie familiale ne sont pas totalement assimilables - et la formulation de la Convention européenne ne doit pas tromper, qui distingue les deux droits. C'est la distinction de ces deux éléments qui a ainsi justifié le fait que la reconnaissance de la vie familiale des couples homosexuels soit si tardive, alors même que leur droit à la vie privée avait été reconnu de longue date $^{2}$. Reste aujourd'hui une interprétation dynamique de l'article $9 \mathrm{du}$ Code civil, qui intègre la vie familiale. Combinée avec l'article 14 du même Code, aux termes duquel «l'étranger jouira en France des mêmes droits civils que ceux qui sont ou seront accordés aux Français par les traités de la nation à laquelle cet étranger appartiendra ", on pourrait en déduire une assimilation entre Français et étrangers s'agissant de la vie familiale. Une analyse plus fine de la situation permet, au contraire, de pointer des différences substantielles et une véritable particularité de la notion de vie familiale appliquée aux étrangers.

Car du fait de la migration qu'elle présuppose, la vie familiale des étrangers présente, on le comprend bien, une double spécificité: une conception originelle d'une part, celle qui existait dans le pays d'origine, et une acception française d'autre part ${ }^{3}$. Le séjour en France étant lié à la seconde, l'étranger n'aura d'autre choix que de s'adapter aux prérequis. Pour autant, les choses ne sont pas toujours si simples, en particulier lorsque la vie familiale a débuté dans le pays d'origine. Deux éléments semblent se dégager pour appréhender cette nécessaire adaptation: d'une part la question des contours de la famille, que les autorités françaises et européennes font varier en fonction des circonstances (I), d'autre part la question des droits attachés au respect de la vie familiale (II).

\section{Les contours de la vie familiale}

La qualité d'étranger sur un territoire d'accueil induit nécessairement un prisme, celui du droit national - et partant, des conceptions - de l'État d'accueil. La notion de famille que l'étranger se verra imposer peut être très similaire à la sienne propre, ou au contraire très éloignée; en toute hypothèse sa réglementation par le droit interne peut varier - deux États ayant des conceptions a priori similaires de la famille peuvent pourtant avoir des règles très différentes, par exemple s'agissant de la filiation adultérine ou des conditions de la fin du mariage. L'étranger va devoir se poser la question de savoir qui, matériellement, est effectivement un membre de sa famille au regard de l'État d'accueil - la France, dans le cas qui nous retiendra ici (A). Cette délimitation de la famille n'est néanmoins que la première marche de l'intégration dans la mesure où la notion de famille va en réalité varier en fonction du droit invoqué (B).

\section{A. Une conception large de la famille}

La question se pose légitimement de savoir qui est dans la famille de l'étranger ${ }^{4}$. L'ordre public international, en France comme ailleurs, vient ici délimiter ce que l'intéressé pourra considérer comme sa famille durant son séjour sur le territoire national.

C'est sans doute la Cour européenne des droits de l'homme qui s'est penchée le plus sur la notion de famille appliquée aux étrangers 5 . Dans un fameux arrêt Marckx $c$. Belgique de 1979, la Cour esquisse une première définition en affirmant que la vie familiale « englobe pour le moins les rapports entre proches parents, lesquels peuvent y jouer un rôle considérable, par exemple entre grandsparents et petits-enfants ${ }^{6}$. La Cour a ainsi développé une acception verticale de la famille, considérant notamment que les relations ascendants / descendants ne sont pas tributaires de l'existence d'un mariage, et ne sont pas impactées par l'éventuelle dissolution de celui-ci ${ }^{7}$ Audelà, les instances européennes ont admis l'acception

1. CESEDA, art. L. 111-1.

2. La Convention EDH a d'abord refusé d'admettre l'existence d'une vie familiale des couples homosexuels (voir, par exemple, Comm. EDH, S. c. Royaume-Uni, 14 mai 1986, req. $\mathrm{n}^{\circ}$ 11716/85), avant de la reconnaitre explicitement (Cour EDH, Schalk et Kopf c. Autriche, 24 juin 2010 , req. ${ }^{\circ}$ 30141/04). En droit interne, la vie familiale des couples homosexuels a été reconnue par le Conseil constitutionnel dans sa décision $2010-92$ QPC du 28 janvier 2011, $M^{\text {mes }}$ Corinne C. et Sophie H.

3. Le Comité des droits de l'homme considère que «la notion de famille peut différer à certains égards d'un État à l'autre, et même d'une région à l'autre à l'intérieur d'un même État, de sorte qu'il n'est pas possible d'en donner une définition uniforme» (Observation générale nº 19, 1990, $\$ 2$ ).

4. La question est notamment posée par D. Vanheule, «Family Reunification of Migrants: Toleration or Right to Stay?», in Les nouveaux Européens. Migration et intégration en Europe, H. Bauer, P. Cruz Villalón, J. Iliopoulos-Strangas (dir.), Bruxelles, É. Bruylant, 2009 , p. 451-476.

5. Voir, parmi d'autres, Le droit au respect de la vie familiale au sens de la Convention européenne des droits de l'homme, F. Sudre (dir.), Bruxelles, Nemesis - É. Bruylant (Droit et justice; 38), 2002.

6. Cour EDH, Marckx c. Belgique, 13 juin 1979, req. $n^{\circ} 6833 / 74, \$ 45$.

7. Voir Cour EDH, Berrehab c. Pays-Bas, 21 juin 1988, req. $\mathrm{n}^{\circ}$ 10730/84, \$21. Le Comité des droits de l'homme a au demeurant développé la même approche: voir Comité des droits de l'homme, Hendricks c. Pays-Bas, 20 décembre 1985, 201/85, \$10.3. 
horizontale de la famille, en retenant les relations entre adultes ou entre collatéraux: ainsi encourage-t-elle le maintien de l'unité des fratries, lorsque les parents se révèlent incapables de continuer à s'occuper de leurs enfants ${ }^{8}$.

Plus avant, on constate l'existence d'une conception large de la famille développée par les instances européennes mais également internes, dès lors qu'est en cause l'éloignement de l'un des membres de la famille. C'est sans doute ici que la conception de vie familiale appliquée aux étrangers développe ses plus larges effets. Le cas de figure est toujours sensiblement le même: un étranger est susceptible d'être éloigné du territoire national, pour une raison administrative ou judiciaire. En France, ce peut être, par exemple, à la suite d'une obligation de quitter le territoire français (OQTF) adoptée par l'autorité préfectorale à la suite d'un refus de titre de séjour, ou encore par un arrêté préfectoral de reconduire à la frontière (APRF) adopté à la suite du prononcé, par le juge répressif, d'une peine d'interdiction du territoire français. Quelle qu'elle soit, la mesure d'éloignement constitue alors une forme d'ingérence dans la vie familiale que l'intéressé a pu développer en France. La question sera inéluctablement la même: l'éloignement de l'étranger est-il réellement compatible avec son droit à une vie familiale? Il s'agit alors, pour l'autorité compétente, de s'interroger sur le nécessaire équilibre entre d'une part les nécessités de l'ordre public, d'autre part le droit individuel de l'étranger. Il lui appartient ainsi d'opérer un contrôle de proportionnalité entre la mesure et le but poursuivi.

La jurisprudence, tant européenne qu'interne, retient ici une conception particulièrement large de la famille, prenant en considération certes le conjoint et les enfants de l'intéressé, mais également le cas échéant le lieu de vie habituel de ses parents et/ ou de ses collatéraux. Dès 1991, l'Assemblée du Conseil d'État, adoptant la droite ligne de la Cour européenne ${ }^{9}$, affirmait qu'il appartient à l'autorité administrative «d'apprécier si, eu égard notamment à la durée et aux conditions de son séjour en France, ainsi qu'à la nature et à l'ancienneté de ses liens familiaux sur le territoire français, l'atteinte que cette mesure porterait à sa vie familiale serait disproportionnée au regard des buts en vue desquels cette décision serait prise ${ }^{10}$. Nombreuses sont les applications jurisprudentielles se fondant sur la vie familiale largement entendue pour venir contrer un éloignement, ou au contraire estimer qu'elles ne sont pas un obstacle à son exécution. Le contrôle opéré par le juge prend alors en considération non seulement la famille (largement entendue) présente en France, mais également celle restée dans le pays d'origine. Un arrêt d'espèce daté du 25 mars 2013 est à cet égard une bonne illustration de cette acception extensive de la famille ${ }^{11}$. Une jeune femme étant l'objet d'une mesure d'éloignement vers son pays d'origine, le juge administratif relève plusieurs éléments permettant de reconnaître l'existence d'une vie familiale en France: le fait qu'elle vivait avec sa mère et sa sœur, qu'elle participait à l'éducation des six enfants de ladite sœur, qu'elle avait un frère, des oncles et tantes en France. On le voit donc, la famille est ici très largement entendue - et permet au Conseil d'État, à rebours de la Cour administrative d'appel, d'estimer que l'éloignement de la requérante vers l'Algérie, pays dans lequel elle n'avait plus qu'une sœur aînée, était une atteinte à sa vie familiale. L'arrêt n'est ni isolé, ni novateur, mais il illustre bien cette acception large ${ }^{12}$.

Pourtant cette approche ne doit pas tromper: car si la famille est extensive dans le cadre de l'examen des mesures d'éloignement, en revanche elle est conçue de façon restrictive s'agissant de l'entrée et du séjour en France.

\section{B. Des conceptions restrictives de la famille}

C'est dans le cadre de deux procédures particulières que la notion de famille sera appréhendée dans un sens curieusement restrictif: d'une part le regroupement familial, d'autre part l'application française du principe de l'unité de famille.

Le regroupement familial est une procédure de droit commun, permettant à un étranger déjà présent sur le territoire français de faire venir sa famille auprès de lui, afin de l'y établir. Le régime est aujourd'hui fondé sur les articles L. 411-1 et suivants du CESEDA, pris en transposition de la Directive relative au regroupement familial, adoptée par l'Union européenne en $2003^{13}$. La procédure est réservée au conjoint majeur et aux enfants mineurs de l'étranger présent en France, à l'exclusion de tout autre membre de sa famille.

S’agissant du conjoint, on constate ici une résilience certaine de cette vénérable institution qu'est le mariage, le conjoint étant appréhendé au sens du droit français ${ }^{14}$. Le mariage doit en outre être conforme à l'ordre public international: la procédure de regroupement familial ne peut concerner qu'un conjoint majeur au jour de la

8. Voir Cour EDH, Olsson c. Suède, 24 mars 1988, req. $\mathrm{n}^{\circ}$ 10465/83.

9. Cour EDH, Moustaquim c. Belgique, 18 février 1991, req. nº 12313/86.

10. CE, Ass., Belgacem et $D^{\text {me }} V^{\text {ve }}$ Babas, 19 avril 1991, Lebon, 1991, p. 152.

11. CE, $M^{\text {le }}$ B., 25 mars 2013, 354837 (inédit).

12. On retrouve au demeurant la même conception sur le plan européen: voir, par exemple, Cour EDH (déc.), Benrachid c. France, 8 décembre 1998, req. $\mathrm{n}^{\circ}$ 39518/98, pour un Algérien vivant en France depuis l'âge de 7 ans, et dont toute la fratrie vit également en France. Dans le cas d'espèce néanmoins il sera relevé que la mesure d'éloignement est légitime du fait, d’une part que le requérant a conservé des liens étroits avec son pays d'origine, dans lequel il a effectué ses obligations militaires, d'autre part que les motifs de son éloignement (plusieurs vols, dont un avec prise d'otage) sont suffisamment graves pour justifier l'atteinte à sa vie familiale.

13. Directive $2003 / 86 / C E$ du 22 septembre 2003 relative au droit au regroupement familial, JOUE, no L. 251,3 octobre 2003 , p. 12.

14. En droit français l'expression "conjoint» vise en effet l'époux/se, à l'exclusion de toute autre forme de couple. Cette acception a été confirmée par le Conseil constitutionnel dans sa décision 2011-155 QPC du 29 juillet 2011, M ${ }^{\text {me }}$ Lejay-Lefebvre. 
demande ${ }^{15}$, et ne peut viser qu'une union qui sera monogamique en France. Ce dernier élément n'est d'ailleurs pas sans soulever de difficultés dans la mesure où, d'une part la solution ne s'est pas toujours imposée, d'autre part elle ne donne aucune règle dans le «choix» du conjoint.

Sur le premier point, il est intéressant de noter que l'union polygamique avait été implicitement admise par le Conseil d'État en $1980^{16}$, l'existence d'une telle union n'étant pas un motif justifiant le refus de régularisation de l'une des épouses et son éloignement du territoire. Les choses ont néanmoins évolué depuis lors: à la suite de la loi du 24 août $1993^{17}$, la situation polygamique sur le territoire français constitue désormais un obstacle dirimant au regroupement familial ${ }^{18}$. Dès lors se pose la question de savoir quel est, précisément, le bénéficiaire du regroupement familial, car ni la loi interne ni le droit de l'Union européenne ne donnent de critère.

Sur ce second point, il importe de relever qu'en soi l'union polygamique ne remet pas en cause l'existence du mariage en lui-même: un étranger ayant plusieurs épouses pourra ainsi faire venir l'une d'entre elles en France, sans que le lien matrimonial ne soit contesté du fait de l'existence d'autres épouses dans le pays d'origine. L'interdiction de la polygamie ne concerne en réalité que le séjour en France. La solution est logique dans la mesure où elle reviendrait, dans le cas contraire, à interdire la polygamie dans le pays d'origine, dès lors que l'époux a l'intention de s'établir en France. Un étranger polygame pourra donc parfaitement voir son union reconnue par la France; mais, justement, «son» union, et non «ses» unions. Il ne pourra faire venir qu'une épouse, et les enfants de celle-ci. Or la difficulté appert dès lors que, pour reprendre le constat dressé par le Conseil d'État lui-même, «les dispositions du Code [...] n'établissent pas de distinction entre le "premier conjoint" d'un étranger polygame et les autres ${ }^{19}$. L'antériorité n'est pas, ici, un critère juridique pertinent. On aboutit ainsi à une situation pour le moins surprenante: la polygamie est en effet rejetée en raison de son inégalité profonde entre les différents conjoints ${ }^{20}$. Or laisser libre choix à l'époux ne peut que renforcer cette inégalité, l'homme ayant ainsi un pouvoir accru sur chacune de ses épouses, qui court le risque d'être abandonnée seule dans le pays d'origine. L'ordre public ainsi conçu, tant par la France que par
l'Union européenne, est certes respectueux des conceptions fondamentales du mariage dans les États d'accueil européens, mais bien peu protectrice des droits individuels des femmes concernées. L'établissement d'un critère unique (antériorité ou autre) serait sans doute peu satisfaisant, mais permettrait au moins de réduire cette toute-puissance de l'homme dans les mariages polygamiques. En revanche, et ici la solution semble plus conforme à un certain souci de protéger les femmes, l'article L. 411-7 du CESEDA n'autorise le regroupement que des seuls enfants de la mère qui pourra elle-même rejoindre son époux. Les enfants issus des autres unions sont, eux, voués à rester auprès de leur propre mère, dans leur pays d'origine. La seule possibilité de les faire venir auprès de leur père sera l'hypothèse dans laquelle leur mère est décédée, ou a été privée de l'autorité parentale à l'issue d'une procédure judiciaire. À défaut d'être satisfaisante eu égard à l'unité de la fratrie, la solution permet de ne pas alourdir encore l'inégalité entre les membres du mariage; elle est en outre conforme à la Convention EDH, car elle constitue une ingérence justifiée dans le droit à une vie familiale garanti par l'article $8^{21}$.

En toute hypothèse, les enfants visés par la procédure de regroupement familial sont, en outre, les enfants mineurs de l'étranger demandeur, ce qui là encore aboutit à une lecture restrictive de la famille: les enfants majeurs au jour de la demande, eux, ne pourront bénéficier de la procédure, et sont dès lors renvoyés au droit commun de l'entrée et du séjour des étrangers en France. De même peut être difficile la venue, au titre du regroupement, de l'enfant accueilli par l'étranger dans sa famille par le biais d'une procédure musulmane de kafala. La kafala a pu être définie comme étant la

[...] décision confiant l'enfant à une famille d'accueil qui assume entièrement son éducation et son entretien, ainsi que la question de ses biens, de manière semblable à une adoption, mais avec la différence importante qu'il ne se produit aucun changement de lien dans la filiation ${ }^{22}$.

Ainsi, faute précisément de ce lien de filiation, la kafala ne peut être assimilée à une adoption - ce que la Cour de cassation rappelle de façon constante ${ }^{23}$. Dans le cadre du regroupement familial, elle aboutit à exclure les enfants ainsi accueillis au sein d'une famille, y compris

15. L'âge de la majorité étant celui reconnu par le droit français (Code civil, art. 414) et non par le pays d'origine, ce que rappelle le Conseil d'État: CE, Ass., GISTI, 29 juin 1990, Lebon, 1990, p. 171.

16. CE, Ministre de l'Intérieur c. $M^{m e}$ Montcho, 11 juillet 198o, Lebon, 1980, p. 315

17. Loi no 93-1027 du 24 août 1993 relative à la maîtrise de l'immigration et aux conditions d'entrée, d'accueil et de séjour des étrangers en France, JORF, 29 août 1993, p. 12196.

18. La prohibition de la polygamie justifie ainsi le refus de renouvellement d'un titre de séjour pourtant obtenu avant l'entrée en vigueur de la loi de 1993: voir CE, GISTI et Association France terre d'asile, 18 juin 1997, Lebon, 1997, tables 651.

19. CE, Ministre de l'Intérieur c. $M^{\text {me }}$ Touré, 2 octobre 2006, Lebon, 2006, tables 897.

20. Voir M. Ancel, "Le statut de la femme du polygame», in Le droit de la famille à l'épreuve des migrations internationales (Actes du colloque du LERADP de Lille II), Paris, LGDJ, 1993, p. 105-124; Comité des droits de l'homme, Observation générale n 28, $2000, \$ 24$.

21. Comm. EDH, A. et A. c. Pays-Bas, 6 janvier 1992, req. nº 14501/89. Pour une application positive en France, voir CE, $M^{m e}$ Sacko, 29 décembre 2000, Lebon, 2000, tables 998.

22. A. Buchner, «La famille en droit international privé», in Recueil des cours de l'Académie de droit international de La Haye, Leyde - Boston, Martinus Nijhoff, 2000, t. 283, p. 118.

23. Voir Cass., $1^{\text {re }}$ civ., 10 octobre 2006, Bulletin civil $I, \mathrm{n}^{\circ} 432$; Cass. $2^{\mathrm{e}}$ civ., 11 juin 2009, Bulletin civil II, $\mathrm{n}^{\circ} 158$; Cass., $1^{\mathrm{re}}$ civ., 15 décembre 2010 , Bulletin civil $I, \mathrm{n}^{\circ}$ 265. La Cour européenne des droits de l'homme a d'ailleurs admis ce rejet de l'assimilation et plus avant considère que, par le 
lorsque l'enfant provient d'un État dans lequel la procédure d'adoption est inconnue et ne peut donc constituer une alternative valable (États de tradition musulmane).

Certes, le droit positif a prévu des aménagements: l'un, posé par l'article L. 411-3 du CESEDA, admet le regroupement familial au profit d'un enfant accueilli par voie de kafala lorsque celle-ci a été prononcée par une autorité judiciaire d'une part, et que l'enfant est celui du conjoint de l'étranger demandeur d'autre part ${ }^{24}$. L'autre aménagement concerne les enfants de nationalité algérienne, du fait de la rédaction de l'Accord franco-algérien de 1968 tel que modifié en $2001^{25}$.

Mais c'est surtout par la voie jurisprudentielle que l'enfant accueilli par kafala a pu retrouver sa place pleine et entière dans le cadre de la vie familiale de l'étranger demandeur d'une procédure de regroupement familial: par un arrêt Sekpon rendu en $2009^{26}$, le Conseil d'État est venu étendre le champ des possibles. En l'espèce, un ressortissant français s'était vu confier la garde d'une petite fille de nationalité béninoise, par le biais d'un acte de kafala judiciaire. M. Sekpon s'était fondé sur ce lien pour demander un visa long séjour au profit de la fillette, ce que le consulat lui avait refusé, motif pris de ce que l'enfant devrait rester auprès des membres de sa famille. Le Conseil d'État a rejeté l'argument, en se fondant sur l'article $3, \$ 1^{\text {er }}$ de la Convention internationale sur les droits de l'enfant (CIDE) et la notion, centrale ici, d'intérêt supérieur de l'enfant - d'effet direct en droit français ${ }^{27}$. Le critère que retient le juge administratif, et que l'on retrouve de façon récurrente depuis lors, est non pas celui d'un lien de filiation (biologique ou adoptive), mais l'exercice de l'autorité parentale. Aux yeux du Conseil d'État, l'intérêt de l'enfant est de demeurer auprès du titulaire de ladite autorité, qui le plus souvent est certes un membre de sa famille, mais peut être un tiers. M. Sekpon est donc fondé à demander un visa au nom de l'intérêt supérieur de l'enfant, et ce même si, précise le Conseil d'État dans un considérant ultérieur, «la délégation d'autorité parentale aurait pour motivation de permettre à la jeune Bellissima Aplogan de s'installer durablement en France». L'invocation de la CIDE, combinée avec l'existence d'une délégation parentale, ouvre ainsi la voie à l'arrivée en France d'enfants accueillis par voie de kafala, y compris s'agissant d'enfants qui ne relèvent pas de l'Accord franco-algérien révisé.

De ce point de vue, il est intéressant de noter que les arrêts relatifs à des enfants algériens invoquent eux aussi, depuis lors, la Convention de 1990, marquant ainsi le caractère plus universel de la solution adoptée. Dans une affaire similaire impliquant une femme française et une enfant algérienne, le Conseil d'État se fonde sur la CIDE pour admettre la venue de la fillette en France ${ }^{28}$; dans ce dernier arrêt, d'ailleurs, la solution est d'autant plus avant-gardiste que l'acte de kafala était notarié et non pas judiciaire. Le juge algérien n'était intervenu que pour autoriser la sortie du territoire de l'enfant confié à la requérante, et non pour entériner la kafala; le juge français, pour sa part, n'y voit pas un obstacle pour la venue de la fillette.

Le Conseil d'État est allé au terme du raisonnement par un arrêt du $1^{\text {er }}$ décembre $2010^{29}$. Le cadre relevait a priori du régime propre aux Algériens: en l'espèce, $M^{\text {me }}$ Hocini, ressortissante algérienne vivant en France, s'était vue confier la garde de la jeune Fatiha, elle-même de nationalité algérienne, par un acte de kafala établi par voie judiciaire, alors que l'enfant avait 9 ans. La requérante avait donc, logiquement, demandé un regroupement familial au profit de la fillette, ce que le préfet du Rhône lui avait refusé. Le Conseil d'État a, lui, enjoint le préfet de délivrer l'autorisation de regroupement, en se fondant sur l'article $3, \$ 1^{\text {er }}$ de la CIDE; l'intérêt supérieur de l'enfant vient, ainsi, atténuer en partie au moins la rigidité de la loi française. Car la solution adoptée par le Conseil d'État ne se fonde pas tant sur l'Accord franco-algérien révisé - et qui par essence ne concerne que les seuls nationaux algériens - que sur la CIDE, Convention qui, elle, a vocation à concerner tous les enfants sans considération de leur nationalité. La position adoptée par le juge administratif étend donc le jeu de la kafala, dès lors que l'intérêt de l'enfant est de vivre avec l'adulte désigné comme le titulaire de l'autorité parentale.

On le constate néanmoins: au-delà des constructions normatives du juge, reste une conception restrictive de la famille dans le cadre de la procédure de regroupement familial - ce qui est d'autant plus important que le respect de cette procédure est le sésame pour bénéficier d'un certain nombre de droits ${ }^{30}$.

jeu des règles du droit international, la France peut légitimement refuser l'adoption d'un enfant accueilli au sein d'un couple par une procédure de kafala: Cour EDH, Harroudj c. France, 4 octobre 2012, req. nº 43631/o9.

24. Cette première exception est néanmoins assouplie lorsque le conjoint n'est pas le parent, mais le tuteur de l'enfant. Voir, en ce sens, CE, Dra et Boulouida, 24 mars 2004 (inédit), concernant des ressortissants marocains. L'épouse du requérant avait été désignée comme tutrice de son neveu, mesure qu'avait confirmée le juge français; le requérant, lui, bénéficiait d'une kafala adoulaire au profit de l'enfant.

25. Voir le titre II modifié, qui vise le conjoint du ressortissant algérien, ses enfants mineurs «ainsi que [1] es enfants de moins de dix-huit ans dont il a juridiquement la charge en vertu d'une décision de l'autorité algérienne».

26. CE, Sekpon, 9 décembre 2009, Lebon, 2009, p. 496.

27. Voir Cass., $1^{\mathrm{re}}$ civ., 18 mai 2005, Bulletin civil I, $\mathrm{n}^{\circ}$ 211; CE, Préfet du Val d'Oise c. $M^{\text {me }}$ Eggley, 7 avril 2006, Lebon, 2006, tables 686. Pour une analyse doctrinale, voir P. Courbe, «L'application directe de la Convention des Nations Unies sur les droits de l'enfant», Dalloz, 2006, Chron. 14987; B. Bonnet, «Le Conseil d'État et la Convention internationale sur les droits de l'enfant à l'heure du bilan. De l'art du pragmatisme», Dalloz, 2010, Chron. 1031.

28. CE, $M^{\text {me }}$ Fournel, 22 octobre 2010, Lebon, 2010, tables 800.

29. CE, $M^{\text {me }}$ Naili ép. Hocini, $1^{\text {er }}$ décembre 2010, 328068.

30. En particulier aux termes de l'article L. 512-2, alinéa 2 du Code de la sécurité sociale, le bénéfice des prestations sociales pour les parents d'enfants étrangers est suspendu au respect de la procédure de regroupement familial. La Cour de cassation avait vu dans cette disposition une mesure fondée sur les nécessités de contrôler les conditions d'accueil des enfants (Cass., Ass. plén., 3 juin 2011, Bulletin, 2011, «Assemblée plénière ", n 5 ). Cette solution est néanmoins nuancée dès lors qu'un engagement international prévoit un traitement identique entre les ressortissants de l'État d'origine et la France: voir, en ce sens, Cass., Ass. plén., 5 avril 2013, 11-18947 et 11-17520, s'agissant respectivement de la Turquie et de l'Algérie. 
On retrouve la même approche dans le cadre de l'unité de famille, principe issu du droit des réfugiés ${ }^{31}$ qui permet à la famille d'un réfugié statutaire de se voir reconnaître, par une sorte de capillarité, la protection internationale fondée sur la Convention de Genève du 28 juillet $1951^{32}$. L'unité de famille est un principe essentiel, dont l'importance a été consacrée dès 1957 par la Commission des recours des réfugiés (CRR) dans une décision Baselga:

[...] la protection que la Convention de Genève [...] a pour objet d'assurer au réfugié serait rendue vaine si elle ne s'étendait pas, non seulement aux enfants mineurs, mais au conjoint du réfugié ${ }^{33}$.

À défaut de pouvoir faire venir auprès de lui les membres de sa famille les plus proches, le réfugié

[...] est placé devant une situation cornélienne: refuser l'asile pour rester uni avec sa famille au risque de s'exposer à la persécution, ou accepter l'offre d'asile en se résignant à l'éclatement de sa famille ${ }^{34}$,

voire à sa mise en danger. Dès lors, il n'est guère concevable, sauf à faire preuve d'un cynisme inacceptable sur le plan humain et difficilement défendable sur le plan juridique, d'exclure de la protection conventionnelle les membres les plus proches du réfugié. La notion de famille est pourtant, ici aussi, conçue de façon restrictive par le droit français. Faute de disposition textuelle explicite, c'est le juge administratif qui s'est trouvé dans l'obligation d'en dessiner les contours. Le Conseil d'État, dans un arrêt d'assemblée Dame Agyepong rendu en décembre 1994, est venu combler le silence des textes. La famille du réfugié est conçue en termes restreints:

Les principes généraux du droit applicables aux réfugiés, résultant notamment des stipulations de la Convention de Genève, imposent, en vue d'assurer pleinement au réfugié la protection prévue par ladite Convention, que la même qualité soit reconnue à la personne de même nationalité qui était unie par le mariage à un réfugié à la date à laquelle celui-ci a demandé son admission au statut, ainsi qu'aux enfants mineurs de ce réfugié ${ }^{35}$.

La notion de famille au sens du droit des réfugiés se rapproche très nettement de celle retenue dans le cadre du droit commun du regroupement familial.
Quelques nuances méritent néanmoins d'être relevées - il est vrai qu'il ne s'agit pas alors «seulement» de leur reconnaître un titre de séjour sur le sol français, mais de leur reconnaître la qualité de réfugié au sens de la Convention de Genève du 28 juillet 1951, ce qui justifie sans doute des préoccupations particulières. Il en résulte que la France a une conception autonome de la famille dans le cadre de l'unité de famille. En particulier le mariage doit être antérieur à la demande du statut de réfugié mais, surtout, les deux conjoints doivent être de même nationalité: à défaut, le juge considère que le couple a toujours la possibilité de trouver asile dans l'État dont est originaire celui des deux conjoints qui n'est pas personnellement persécuté. Pour autant, la solution a dû être précisée par le Conseil d'État. Dans un premier temps, l'existence d'une nationalité en commun était suffisante, et justifiait la reconnaissance du statut au titre de l'unité de famille: dans une affaire Boularouf, le juge du fond avait ainsi admis d'appliquer le principe à un conjoint ayant la double nationalité russe et marocaine, et dont l'épouse russe était réfugiée statutaire ${ }^{36}$. Cette analyse a néanmoins été cassée par le Conseil d'État: la Haute Juridiction précise, en 2009, que M. Boularouf pouvant se prévaloir de la protection du Maroc, il ne peut bénéficier du principe de l'unité de famille ${ }^{37}$. Dès lors, les deux conjoints doivent avoir la même nationalité, à l'exclusion de toute autre; à défaut, il faudra prouver pour chacun l'existence de craintes personnelles de persécution dans leur propre État ${ }^{38}$.

Le principe de l'unité de famille issu de l'arrêt Agyepong s'étend en outre aux enfants mineurs du réfugié, ce qui exclut tout à la fois les enfants accueillis par voie de kafala (faute d'un lien de filiation) et ceux du conjoint du réfugié statutaire. La conception française du principe peut ainsi aboutir à l'éclatement de familles, soit parce que l'enfant est issu d'une précédente union et n'a pas été adopté par le réfugié, soit parce qu'il est majeur.

Pour autant, il importe de noter que la conception de la famille a pu être élargie par la jurisprudence dans le cadre de la protection des personnes placées sous tutelle. S'il est de jurisprudence constante que ni les ascendants ni les collatéraux ne peuvent relever de la famille au sens de l'unité de famille, une nuance apparaît dès lors qu'a été prononcée une mesure de tutelle. Dès 1999, la Commission

31. Ce qui permet d'en exclure le bénéfice aux étrangers déboutés du statut de réfugié mais qui obtiennent la protection subsidiaire fondée sur l'article L. 712-1 du CESEDA : voir CE, OFPRA c. M'me Ananian ép. Arakelian, 18 décembre 2008, Lebon, 2008 , tables 775.

32. Convention portant statut international des réfugiés, Genève, 28 juillet 1951, Recueil des traités des Nations unies, vol. 189 , p. 150.

33. CRR, Baselga, 12 mars 1957, 1474, in Jurisprudence de la Commission de recours des réfugiés, préface de R. Cassin, introduction de A. Heilbronner, Paris, Dalloz, 1961, p. 93.

34. R. Ergec, «Le Conseil de l'Europe et les réfugiés», in La reconnaissance de la qualité de réfugié et l'octroi de l'asile (Actes de la journée d'études du 21 avril 1989 organisée par l'Université libre de Bruxelles, Centre de droit international de l'Institut de sociologie, Centre de droit public de la Faculté de droit), Bruxelles, É. Bruylant, 1989, p. 128.

35. CE, Ass., Dame Agyepong, 2 décembre 1994, Lebon, 1994, p. 523, avec les conclusions de M. Denis-Linton.

36. CRR-SR, Boularouf, 27 mai 2005

37. CE, OFPRA c. M. Boularouf, 23 février 2009, Lebon, 2009, p. 64.

38. Ce qui peut alors poser une difficulté particulière, lorsque l'étranger est polygame et que chacun des conjoints se voit reconnaitre le titre de réfugié à titre personnel, et non sur le fondement de l'unité de famille. Le principe est alors celui de l'indépendance des situations. Néanmoins, le réfugié se voit refuser la carte de résident (prohibée dans le cadre de la polygamie depuis 1993), mais peut obtenir une carte de séjour temporaire. Voir ici CAA Lyon, Pang Cheng Xiong, 29 septembre 2005, ooLY01472. 
des recours des réfugiés admet qu'une ressortissante camerounaise trisomique, placée sous la tutelle de son oncle lui-même réfugié statutaire en France, puisse bénéficier du principe de l'unité de famille ${ }^{39}$. Par un arrêt de 2004, le Conseil d'État a repris le même raisonnement au profit des ascendants ${ }^{40}$. Deux conditions sont alors exigées: d'une part la situation de particulière dépendance doit avoir existé dans le pays d'origine, d'autre part elle doit être concrétisée par une mesure de tutelle constatée par un juge français. Sous ces deux limites néanmoins, l'unité de famille peut être étendue à d'autres membres de la famille que ceux explicitement visés par l'arrêt de 1994, ce qui aboutit à un assouplissement des conditions restrictives.

Le constat est néanmoins très ambigu : la famille des étrangers est définitivement protéiforme, ses contours évoluant selon des circonvolutions administratives qu'il peut être difficile d'appréhender pour le non-spécialiste, à plus forte raison pour l'étranger lui-même. L'enjeu est pourtant de taille, en raison des droits qui sont attachés à la vie familiale.

\section{Les droits attachés à la vie familiale de l'étranger}

Nonobstant la relativité de la conception de la famille des étrangers dans le droit français, ce dernier attache à celle-ci des conséquences majeures. Deux semblent particulièrement intéressants : d'une part la question du séjour de l'étranger fondée sur sa vie familiale (A), d'autre part l'émergence, depuis une trentaine d'années, du concept de «vie familiale normale» $(B)$.

\section{A. Le lieu de la vie familiale}

Si l'étranger a indéniablement le droit à une vie familiale, ce droit ne se confond pas avec le droit au séjour. La Cour européenne des droits de l'homme le rappelle régulièrement, «l'article 8 ne saurait s'interpréter comme comportant pour un État contractant l'obligation générale de respecter le choix» par des étrangers du lieu de leur installation ${ }^{41}$. L'État est donc en droit de refuser une autorisation de regroupement familial dès lors que les intéressés ont la possibilité de mener une vie normale dans le pays d'origine - et ce au nom du principe selon lequel il échet aux États de contrôler leurs frontières, sous réserve de leurs engagements internationaux, y inclus le droit à une vie familiale garanti par l'article 8 de la Convention $\mathrm{EDH}^{42}$. Au-delà du nécessaire contrôle de proportionnalité entre contrôle de l'immigration et droit à une vie familiale, l'État se doit de préserver, autant que possible, l'unité familiale, quitte à ce que cette unité s'établisse en dehors du territoire national. Ainsi, dans un arrêt Mubilanzila Mayeka et autres c. Belgique rendu en $2010^{43}$, la Cour EDH affirme que l'expulsion du requérant ne brise pas la vie familiale dès lors que la révocation de son titre de séjour conduit à la révocation de ceux de son épouse et de son fils - sous réserve de ce, qu'effectivement, la vie familiale est possible dans des conditions normales dans le pays d'origine ${ }^{44}$.

Cette jurisprudence reste limitée néanmoins aux hypothèses dans lesquelles l'étranger et sa famille pourraient effectivement mener une vie familiale normale dans le pays d'origine ${ }^{45}$. Elle ne doit pas aboutir à briser la vie familiale menée dans l'État d'accueil, ainsi que le rappelle la Cour européenne dans un arrêt Şen c. Pays-Bas de $2001^{46}$. Tel sera le cas lorsque, comme dans l'affaire de 2001, l'étranger a certes laissé un enfant dans le pays d'origine, enfant qui y est né et y a toujours vécu, mais qu'il a par ailleurs d'autres enfants nés et scolarisés dans l'État d'accueil. Interdire le regroupement au profit de l'enfant resté dans le pays d'origine aboutit alors à violer le droit à une vie familiale, en plaçant les parents dans l'obligation soit de renoncer à vivre avec l'un de leurs enfants, soit de contraindre leurs autres enfants à un déracinement, en s'établissant dans un pays que les cadets n'ont jamais connu. L'intérêt supérieur de l'enfant, garanti par la Convention internationale sur les droits de l'enfant (art. $3, \$ 1^{\mathrm{er}}$ ), vient alors nuancer le jeu de la solution.

C'est d'ailleurs cette même notion qui peut, dans certaines hypothèses ciblées, venir, au nom de la vie familiale, fonder un véritable droit au séjour. La problématique est alors celle des anchor children, dans laquelle un enfant ancre véritablement son ou ses parents sur le territoire de l'État d'accueil. L'enfant a un droit de résidence permanent sur le territoire - en règle générale du fait qu'il a la nationalité de l'État en cause. La question se pose de savoir quel sort réserver à ses parents, eux-mêmes étrangers (en situation régulière ou non). Le cas peut se présenter régulièrement dans un État dont l'attribution de nationalité est régie par le ius soli simple; il peut également survenir en France, par exemple lorsque l'un des parents est certes français, mais l'autre étranger en situation irrégulière, et sans qu'il y ait forcément de communauté de vie entre les

39. CRR, Ngambi, 6 mai 1999.

40. CE, $M^{\text {me }}$ Trin ép. Mer, 28 juillet 2004, Lebon, 2004, p. 353, L'actualité juridique. Droit administratif, 2004, p. 1874, conclusions F. Donnat.

41. Cour EDH, Abdulaziz, Cabales et Balkandali c. Royaume-Uni, 28 mai 1985, req. nº 9214/80, $\$ 68$.

42. Cour EDH, Beldjoudi c. France, 26 mars 1992, req. $n^{\circ} 12083 / 86, \$ 74$.

43. Cour EDH, Mawaka c. Pays-Bas, $1^{\text {er }}$ juin 2010, req. n $29031 / 04$.

44. Cour EDH, $M^{m e}$ Mubilanzila Mayeka et $M^{\text {He }}$ Tabitha Kaniki Mitunga c. Belgique, 12 octobre 2006, req. $\mathrm{n}^{\circ}$ 13178/o3, $\$ 81$ sq.

45. Pour une application par le droit interne français, voir, parmi d'autres, CE, Zhang, 13 juin 2012 : le Conseil d'État relève que nonobstant le fait que deux des trois enfants du requérant sont nés en France, aucun obstacle n'existe pour que l'ensemble de la famille ne puisse mener une vie normale dans le pays d'origine, en l'espèce la République de Chine. Le Conseil d'État note en particulier que les enfants pourront y être scolarisés.

46. Cour EDH, Şen c. Pays-Bas, 21 décembre 2001, req. nº 31465/96. 
deux parents. L'État d'accueil peut être tenté d'éloigner les parents, sans pour autant imposer en droit l'éloignement de l'enfant; l'unité familiale induit, néanmoins, qu'en pratique l'enfant sera, lui aussi, éloigné d'un territoire dont pourtant il a la nationalité.

La Cour de justice a été saisie de la question à travers l'affaire Zhu et Chen de $2004^{47}$. En l'espèce, une ressortissante chinoise, déjà mère d'un enfant lui-même chinois, avait donné naissance à Belfast à une petite fille. Par application de la loi irlandaise (Citizenship Act, 1956), la fillette s'était vue attribuer la nationalité irlandaise du fait de sa naissance. La mère, en son nom propre et au nom de sa fille, avait sollicité un titre de séjour longue durée sur le territoire britannique, demande qui avait été refusée par les autorités compétentes. Saisie par le Secretary of State of the Home Department, la Cour de Luxembourg a ainsi dû prendre position sur la question de savoir si le ressortissant d'un État tiers à l'Union européenne peut revendiquer un titre de séjour longue durée du fait que son enfant est, lui, un citoyen de l'Union ${ }^{4}$. L'argumentation du gouvernement britannique était simple: dès lors que $\mathrm{M}^{\mathrm{me}}$ Chen admettait que son déplacement avait eu pour seule finalité de permettre à son enfant d'acquérir la nationalité de l'un des États membres, elle ne pouvait se prévaloir des effets de la nationalité. Le juge de l'Union rejette cette vision des choses: rappelant que l'attribution de nationalité est une compétence exclusive des États ${ }^{49}$, elle considère que la seule question qui se pose à elle est celle de savoir si ladite nationalité peut avoir une incidence sur le séjour de ses ascendants. Et sa réponse est claire: au nom de la libre circulation des citoyens de l'Union, et en considération de l'intérêt supérieur de l'enfant, la mère, ressortissante d'un État tiers, doit elle-même avoir un droit au séjour. Il est intéressant de noter que l'argumentation retenue par la Cour se fonde sur l'idée d'effet utile du principe de libre circulation: à défaut de permettre à la mère de s'installer durablement sur le territoire de l'un des États membres, le droit reconnu au citoyen de l'Union serait vidé de tout sens faute de pouvoir raisonnablement être mis en ouvre lorsque le citoyen est mineur.

La solution a été reprise par la Cour européenne des droits de l'homme en 2006, dans une affaire Rodrigues Da Silva c. Pays-Bas ${ }^{50}$. La Cour de Strasbourg a néanmoins une conception plus élargie encore que le juge de l'Union, admettant le jeu de la règle y compris lorsque la mère est en séjour irrégulier sur le territoire de l'Union. En l'affaire, une ressortissante brésilienne avait donné naissance à une petite fille, de nationalité néerlandaise du fait de son père. Le couple avait vécu quelque temps en commun avant de se séparer; à aucun moment la mère, en situation irrégulière eu égard à la loi néerlandaise, n'avait sollicité la régularisation de sa situation. Ce n'est que six années plus tard qu'une telle demande fut introduite - demande rejetée par les autorités. Rappelant sa jurisprudence Moustaquim de 1991 et le nécessaire juste équilibre entre le droit à une vie familiale et les nécessités de contrôler l'entrée et le séjour des étrangers, la Cour EDH constate l'existence de liens étroits entre la mère et la fillette ${ }^{51}$. Elle considère qu' «il est manifestement dans l'intérêt de cette dernière que sa mère demeure aux Pays-Bas» et en déduit que, "dans les circonstances de l'espèce», il y a violation de l'article $8^{52}$.

On le constate, la Cour de Strasbourg est donc prudente, se refusant à ériger en principe absolu un cas particulier; pour autant elle s'inscrit dans la continuité de la jurisprudence de la Cour de justice de l'Union. Surtout, elle consacre le cas particulier qu'est, eu égard aux principes dégagés, la question des parents de citoyens mineurs de l'Union. Il importe néanmoins de noter une nuance: dans l'affaire $Z h u$ et Chen, l'enfant était âgée de quelques mois - la Cour de justice le souligne, au demeurant; dans l'affaire Rodrigues Da Silva, elle n'avait que dix ans au moment de l'arrêt de la Cour. On pourrait donc être tenté de considérer que la solution reste limitée, d'autant que, dans ces affaires, le citoyen de l'Union est à la charge du parent ressortissant d'État tiers. La solution a néanmoins été élargie à l'hypothèse inverse: selon l'arrêt Jia de janvier de $2007^{53}$, l'ascendant qui est à la charge du citoyen européen peut lui-même obtenir un titre de séjour sur le territoire de l'Union - solution confirmée l'année suivante dans un arrêt Metock et autres ${ }^{54}$, s'agissant du droit des conjoints.

\section{B. La normalité de la vie familiale}

Au-delà du séjour éventuel, apparaît une autre interrogation: celle des conditions d'exercice de la vie familiale de l'étranger. Dans un arrêt GISTI daté de $1978^{55}$, le Conseil d'État a dégagé un principe nouveau: celui de «vie familiale normale», notion qu'il ne juge pas pour autant utile de définir avec précision. Le cœur du problème posé par ce nouveau concept n'est en fait pas tant la vie familiale, que sa normalité. Le Conseil d'État ne la délimite pas, pas plus

47. CJ, Ass. plén., Catherine Zhu et Lavette Chen, 19 octobre 2004, C-200/02.

48. Le lien de rattachement avec une situation permettant la mise en œuvre avec le droit de l'Union était ici assuré par le fait que $\mathrm{M}^{\mathrm{me}} \mathrm{Chen}$ et sa fille avaient sollicité un titre de séjour en Grande-Bretagne, et non en Irlande. Sur l'obligation d'avoir un tel lien, voir CJ, Morson et Jhanjan, 27 octobre 1982, C-35 et 36/82 (mères surinamaises de ressortissants néerlandais n'ayant jamais fait valoir leur liberté de circulation sur le territoire de l'Union); CJ, Carpenter, 11 juillet 2002, C-60/oo (épouse philippine d'un ressortissant britannique, en situation irrégulière).

49. CJ, Ass. plén., Catherine Zhu et Lavette Chen, 19 octobre 2004, pt. 37.

50. Cour EDH, Rodrigues Da Silva et Hoogkamer c. Pays-Bas, 31 janvier 2006, req. n 50435/99.

51. Ibid., $\$ 35$.

52. Ibid., $\$ 37$

53. CJ, Jia, 9 janvier 2007, C-1/05.

54. CJ, Blaise Metock et autres c. Minister for Justice, 25 juillet 2008, C-127/08.

55. CE, Ass., GISTI, CFDT et CGT, 8 décembre 1978, Lebon, 1978, p. 493. 
que ne le fait d'ailleurs le Conseil constitutionnel lorsqu'en 1997 il relève l'existence du droit de mener une vie familiale normale ${ }^{56}$, ou encore en 2003 dans une décision rendue en matière d'asile, il évoque la possibilité, pour l'étranger, de «mener une existence normale» dans son pays d'origine ${ }^{57}$. Dans le cadre du contentieux de 1978, néanmoins, la notion induit tout à la fois le droit au regroupement familial et le droit, pour ces membres de la famille venus dans le cadre d'une procédure régulière, d'occuper un emploi. L'idée de vie familiale normale semble ainsi renvoyer à l'idée d'un niveau de vie suffisant ${ }^{58}$, et partant supérieur au seuil de pauvreté en France. L'accès à l'emploi serait un élément constitutif de la normalité.

Cette acception correspond de surcroît à la jurisprudence plus récente de la Cour de justice. Dans un arrêt de Grande Chambre Ruiz Zambrano de 2011, la juridiction de l'Union européenne s'est penchée sur la question sus-évoquée des ascendants de citoyens de l'Union 59. Reprenant la jurisprudence précitée, la Cour précise que l'ascendant d'un citoyen de l'Union doit se voir reconnaitre non seulement le droit au séjour dans l'État de nationalité de son enfant, mais qu'en outre il doit s'y voir accorder un permis de travail, sous peine de "priv[er] lesdits enfants de la jouissance effective de l'essentiel des droits attachés au statut de citoyen de l'Union ${ }^{60}$. La solution semble dès lors fixée, tant par les juridictions internes qu'européennes: le droit à une vie familiale normale induit ainsi les deux éléments que sont d'une part le principe de l'unité de la famille, d'autre part la possibilité, par le travail, de vivre dans des conditions normales. C'est sans doute ainsi que doit être comprise la vie familiale des étrangers, nonobstant son hétérogénéité administrative. 